\section{Three subroutines for dealing efficiently with permutations*}

\author{
WILLIAM P. DUNLAP \\ Tulane University, New Orleans, Louisiana 70118
}

Subroutines for generating and indexing permutations are described. The first generates a permutation of $N$ integers, depending on the value of an index that may take on values between 1 and $N$ !, the number of possible permutations of length $\mathbf{N}$. The second subroutine calculates an index from a given permutation that denotes its position in the list of all possible permutations of that length. The last subroutine generates random permutations of any given length.

The usefulness of permutations in experimental design has long been recognized. Cochran and $\operatorname{Cox}$ (1957) outlined procedures for the construction of random orderings of integers by hand using a table of random numbers; tables of random permutations are available (e.g., Fisher \& Yates, 1963; Moses \& Oakford, 1963). Random permutations are clearly more convenient than random numbers for assigning Ss to treatment groups, since this operation ordinarily involves sampling randomly without replacement from a fixed pool of Ss to produce groups of equal size. The fact that $\mathrm{Ss}$ are so assigned to groups provides the basis for a class of nonparametric techniques known as randomization tests (Edgington, 1969), which involve the use of permutations. Permutations are also used to match groups, to counterbalance treatments, and to specify the order of test materials such

Table 1

SUBROUTINE PERIN (K,N, IND)

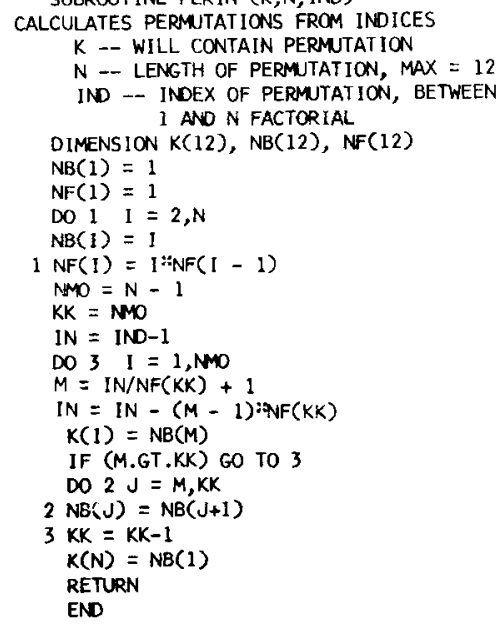

* Requests for reprints should be sent to William P. Dunlap, Department of Psychology, Tulane University, New Orleans, Louisiana 70118. as number or letter sequences. Handling permutations with computers can be costly both in terms of programming and execution time; the following subroutines have been found useful and efficient on both counts.

Table 1 presents the FORTRAN code for subroutine PERIN, which generates a permutation of the integers

Table 2

The Relationship Between the Index, IND, and the Permutations of Length 3

\begin{tabular}{cccc}
\hline IND & \multicolumn{3}{c}{ Permutation of Length 3} \\
\hline 1 & 1 & 2 & 3 \\
2 & 1 & 3 & 2 \\
3 & 2 & 1 & 3 \\
4 & 2 & 3 & 1 \\
5 & 3 & 1 & 2 \\
6 & 3 & 2 & 1 \\
\hline
\end{tabular}

1 to $N$, leaves the result in Array $K$, and depends on the index, IND, which is supplied by the user and may range between 1 and $\mathrm{N}$ factorial. If PERIN is called repeatedly with IND taking on successive values from 1 to $N$ !, the entire ordered list of permutations of Size N will be generated; for an example with permutations of Size 3 , see Table 2. If IND is given random values between 1 and $N$ !, PERIN will generate a list of random permutations of Length $N$. Unfortunately, the exact specification of permutations in this manner is limited by the word size of the computer involved, since $N$ ! must be smaller than the maximum value an integer may take on. 1

Subroutine INPER, listed in Table 3 , calculates the index, IND, that corresponds to the particular permutation of Length $N$ present in a particular ordering of the integers 1 through $N$ in Array $K$. IND is found to be 1 if the integers are in perfect ascending order, and $\mathrm{N}$ ! for the reverse order. Between these limits, IND takes on values in accordance to an algorithm in which right-handmost Array $\mathrm{K}$. The user must supply $\mathbf{N}$ and
SUBROUTINE INPER $(X, N$, IND)

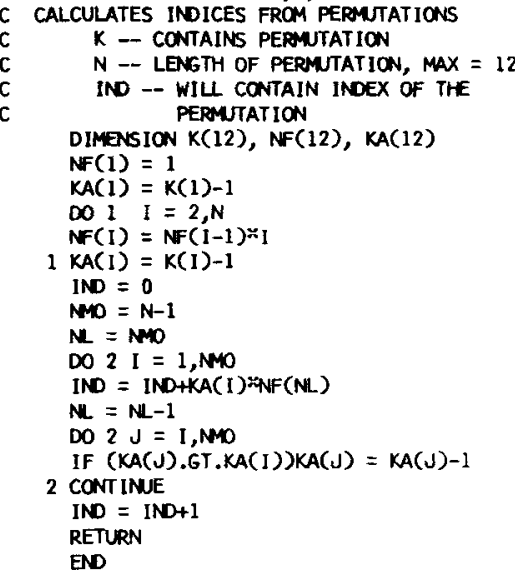

digits change position most frequently. Being able to specify and catalog permutations in this manner makes it easy to do frequency counts of permutations that might occur as data; also, it simplifies the specification of a given permutation. Again, permutation length is limited by computer word size.

Since one of the most important uses of permutations is randomization, and since the previous subroutines are limited in length by computer word size, a third subroutine that produces random permutations of any given length has been found quite useful. Subroutine RANPER, listed in Table 4, incorporates a modification of the pseudorandom number generator, RANDU, from IBM's (1969) Scientific Subroutine Package. The constants in RANDU depend upon computer word length; the present constants are for 32-bit words, such as those used by the IBM 360 . In

Table 4

SUBROUTINE RANPER (K,N, IY) C CALCULATES RANDOM PERMUTATIONS K -- WILL CONTAIN PERMUTATION $N$-- LENGTH OF PERMUTATION

IY -- INITIALLY MUST BE SET EQUAL TO A 7 - 9 DIGIT ODD INTEGER

DIMENSION K(1000)

DO I I $=1, N$

$1 K(1)=1$

$\mathrm{NM}=\mathrm{N}-1$

$N L=N$

$\mathrm{FL}=\mathrm{NL}$

DO $51=1, N M$

C GENERATE PSELDO RANDOM NMMBER IY $=I Y: 65539$

IF (IY) $2,3,3$

2 IY $=I Y+2147483647+1$

$3 \mathrm{YFL}=I Y$

$I R=Y F L * 465661 E-9 * F L+1.0$

C REORDER ARRAY $K$

$I S=K(I R)$

$N L=N L-1$

$\mathrm{FL}=\mathrm{NL}$

IF (IR.GT.NL) GO TO 5

DO $4 \mathrm{~J}=\mathrm{IR}, \mathrm{NL}$

$4 K(J)=K(J+1)$

$5 K(N L+1)=I S$

RETURN

END 
the main routine, the user must supply a 7- to 9-digit odd integer as an initial value for IY to start the random number generator; thereafter, IY is set equal to the last random integer produced. The user must also specify $N$, the length of the permutation desired. The usefulness of this last program is easily seen when one considers a problem such as randomly ordering the position of 60 rats on a cage rack. With a random number table, selection of a random order is arduous to say the least, and tables of permutations of Length 60 are not readily available. $A$ single random permutation generated by RANPER does this job quite nicely.

$$
\text { REFERENCES }
$$

COCHRAN, W. G., \& COX, G. M. Experimental designs. (2nd ed.) New York: Wiley, 1957.

EDGINGTON, E. S. Statistical inference: The distribution-free approach. New York: McGraw-Hill, 1969.

FISHER, R. A., \& YATES, F. Statistical tables for biological, agricultural and medical research. New York: Hafner, 1963.

IBM. Scientific subroutine package. White Plains, N.Y: International Business Machines Corp., 1969, Manual H 20-0205-03.

MOSES, L. E., \& OAKFORD, R. V. Tables of random permutations. Stanford, Calif: Stanford University Press, 1963. NOTE

1. For computers with a standard 32-bit word, such as the IBM 360 , the maximum value an integer can have is $2^{31}-1$. The largest factorial less than this value is 12 ! thus limiting permutation length to 12. With a 36-bit word, the maximum length is 13 . 